\title{
Trigger Warnings: Caring or Coddling
}

\author{
Helen M. Stallman \\ School of Psychology, Social Work and Social Policy \\ University of South Australia \\ International Association for University Student Health and Wellbeing \\ Diann S. Eley \\ Faculty of Medicine \\ The University of Queensland \\ International Association for University Student Health and Wellbeing \\ Amanda D. Hutchinson \\ School of Psychology, Social Work and Social Policy \\ University of South Australia
}

\begin{abstract}
Trigger warnings are proposed as a strategy to keep university students safe from potentially distressing material. While used internationally for several years, they have begun to be formally implemented in some Australian universities. This paper provides a critical review of the underlying theoretical premises of trigger warnings and the personal and professional impact of such warnings for students in health professions. As with most short-term avoidance strategies, trigger warnings are likely to have a negative impact on student mental health, rather than improve it. The warnings undermine the autonomy and selfmanagement skills of students as independent, adult learners. If these warnings become more widespread, they have significant implications for the resilience of students. For students in health professions, who need skills to manage a wide-range of often unpleasant topics and provide responsive and competent treatment for their patients, they have the potential to result in adverse outcomes in patient care. The development of coping skills to flexibly self-manage and self-regulate emotions and behaviour is an important graduate attribute and provides an alternative to counterproductive trigger warnings.
\end{abstract}

Keywords: coping, mental health, resilience, trigger warnings, wellbeing;

\section{Trigger warnings: Caring or coddling}

Universities aim to provide a safe and healthy environment for their students, staff and visitors. However, some universities internationally and now in Australia (Palmer 2017), have taken this further to introduce trigger warnings into their policies and practices to provide a safe inclusive space for students (NOWSA 2017). A trigger warning is a statement at the start of a lecture, piece of writing, or resource alerting the audience that it contains potentially distressing material. These include courses where there may be discussion of sexual assault, violence, domestic abuse, child abuse, eating disorders, self-harm, suicide, pornography, abortion, kidnapping, hate speech, animal cruelty, animal deaths, or abattoirs. The more extensive list of the Network of Women Students Australia (NOWSA 2017) suggests that some adult learners cannot cope without prior warning of: classism, corpses, skulls or skeletons, drug use or talk of drugs (legal, illegal or psychiatric), eye contact, food, gore, insects, medical procedures, mental illness, needles, panic attacks, pregnancy, slimy things, snakes, spiders, trichotillomania, vomit, warfare or weapons. The aim of this commentary is to highlight the implications of such warnings for students in health profession programs.

The experience of a wide-range of emotions is part of being human - from distress through to euphoria. Emotions are not triggered by external events, but by an individual's thoughts about those events (Beck 2002). These in turn are influenced by a person's psychosocial history. Humans develop coping skills to manage their psychological and social environments. Some are healthy; 
such as coping self-talk, exercising, talking to a trusted friend or family member, or a health professional; some are unhealthy such as eating, drinking alcohol, or social withdrawal (Stallman and Wilson 2017). In the case of anxiety, without an actual threat, flight or fight is an unhealthy strategy (e.g. Boyes 2015).

Trigger warnings are likely to increase anxiety rather than promote coping and resilience, because they send a message to all students that the environment needs to protect them because they cannot cope independently. It suggests students need warnings of potentially unpleasant material because students do not have the coping skills to manage the unexpectedness that happens in life or even in a considered environment such as a university course they enrolled in. Trigger warnings, by definition, suggest that students need to be explicitly warned of content because the students: 1) cannot select programs of study based on their individual strengths and vulnerabilities; 2) are unable to anticipate the types of content that may be covered within a course, based on the program they have chosen to study; 3) they have inadequate coping skills and may therefore be harmed by exposure to course content; and 4) they need to avoid exposure to unpleasant things or something catastrophic may happen.

Some people who experience traumatic experiences go on to develop a range of psychopathology, including depressive and anxiety illnesses. It is important that these people receive treatment to help them overcome or at least manage their symptoms (National Institute of Clinical Excellence 2005). Left untreated, these illnesses can result in increased unhealthy avoidance and social withdrawal that can perpetuate or exacerbate symptoms, rather than help people manage their illnesses. Students who are unable to implement healthy coping strategies independently should work with a mental health professional to help them decide what activities they are able to do - this may include not enrolling in particular programs or courses while they are unwell.

In contrast, universities provide a learning environment for adult education. At this tertiary level of education, it is presumed that students have sufficient personal coping strategies to effectively interact with a diversity of people, learn content relevant to the program of study they choose to enrol in, and tolerate diverse and often contradictory opinions of others. Indeed, university is an optimal environment to be confronted, be uncomfortable, and grow by thinking about topics in a new way. To safeguard those students who may not cope well with these situations, most universities provide free counselling, disability, and General Practice services for individual support. This approach is consistent with promoting student autonomy and self-efficacy by giving students the responsibility to: 1) choose the timing of their studies after taking into account competing priorities in their life; 2) engage in studies that are consistent with their strengths and vulnerabilities, including current known mental illnesses; and 3) cope. Adult education recognises the ability of students to regulate their own emotions, and to choose if, and when, to seek additional support without impeding autonomy by coddling.

If these triggers warnings become more widespread, they have serious implications for students in health professions who are likely exposed to many of these 'trigger' topics in the course of their studies and work with unwell people. While an Arts student who seeks to be protected from literature they find uncomfortable will only narrow their individual learning, the student in a health program who seeks to be protected from any potentially distressing content will be ill-prepared to respond flexibly and with empathy and confidence to situations they may encounter as a health professional. This is likely to impact patient care and has the potential for adverse patient outcomes.

Resilience develops from experiencing distress, coping with it, and learning from the experience. Warning students about exposure to potentially distressing material may prevent them from engaging with unpleasant material and from successfully managing their distress. The nature of the 
practice of health professions means that training will be full of confronting and often unpleasant concepts, situations, and experiences. Coping with challenges flexibly is a vital skill for health professionals-as important as their ability to problem-solve, think critically, and engage in competent and professional practice. Engagement with challenging material is likely to benefit the student and their future patients (Heath, et al. 2017) through the development of resilience, responsibility, and resolve (Eley and Stallman 2014). The University, with its support services, is the ideal setting for this autonomous learning and emotional regulation to occur. In contrast to trigger warnings, it has been suggested that teaching coping planning to students will better prepare them for life as a professional and in general (Stallman \& Wilson 2017). It is important to promote university student mental health (Stallman 2010). The solution to improving mental health, however, is not to coddle students further, but to encourage and support their coping skills and resilience (Stallman \& Wilson 2017). 


\section{References}

Beck, A. T. (2002). Cognitive models of depression. In R. L. Leahy and E. T. Dowd (Eds.) Clinical advances in cognitive psychotherapy: Theory and application, (pp 29-61). New York, Springer.

Boyes, A. (2015). The anxiety toolkit: Strategies for fine-tuning your mind and moving past your stuck points. TarcherPerigee.

Eley, D. and H. M. Stallman (2014). Where does medical education stand in nurturing the 3 Rs in medical students: Responsibility, resilience, and resolve? Medical Teacher 36(10), 835-837.

Heath, M., et al. (2017). Teaching sensitive material: A multi-disciplinary perspective. ERGO.

National Institute of Clinical Excellence (2005). Post-traumatic stress disorder: management. Clinical guideline [CG26]. Available at nice.org.uk/guidance/cg26, Author.

NOWSA (2017). Trigger warning policy; Retrieved from https://nowsa2017.com/trigger-warning-policy-2/.

Palmer, T. (2017). Monash University trigger warning policy fires up free speech debate. ABC News. http//www.abc.net.au/news/2017-03-28/monash-university-adopts-trigger-warning-policy/8390264.

Stallman, H. M. (2010). Psychological distress in university students: A comparison with general population data. Australian Psychologist 45(4), 249-257.

Stallman, H. M. (2017). Coping planning: A patient- and strengths-focused approach to suicide prevention. Australasian Psychiatry

Stallman, H. M., \& Wilson, C. (2017). Could explicit teaching coping planning for suicide prevention improve resilience in medical students? Medical Teacher, 39(7), 680. doi:http://dx.doi.org/10.1080/0142159X.2017.1302574

The authors may be contacted via

helen.stallman@unisa.edu.au 